Acta Univ. Sapientiae, Informatica 11, 2 (2019) 142-158

DOI: 10.2478 /ausi-2019-0010

\title{
Evolutionary solving of the debts' clearing problem
}

\author{
Csaba PĂTCAȘ \\ Babeș-Bolyai University \\ Cluj-Napoca, Romania \\ email: patcas@cs.ubbcluj.ro
}

\author{
Attila BARTHA \\ Babeș-Bolyai University \\ Cluj-Napoca, Romania \\ email: abartha@yahoo.com
}

\begin{abstract}
The debts' clearing problem is about clearing all the debts in a group of $n$ entities (banks, companies etc.) using a minimal number of money transaction operations. The problem is known to be NP-hard in the strong sense. As for many intractable problems, techniques from the field of artificial intelligence are useful in finding solutions close to optimum for large inputs. An evolutionary algorithm for solving the debts' clearing problem is proposed.
\end{abstract}

\section{Introduction}

The problem of debt clearing (DC problem) can arise among a group of friends, but it also needs to be solved regularly among the affiliates of multinational corporations, banks or even countries $([16,18])$. As money transactions are time- and money-sensitive operations, it can be desirable to clear the debts in a minimal number of money transaction operations.

Problems related to debt clearing were studied in the past. Shapiro gave a linear programming based model to minimize the cost of payments netting assuming that costs are directly proportional to the volume transacted ([16]).

Computing Classification System 1998: I.2.8

Mathematics Subject Classification 2010: 68T20

Key words and phrases: debt settling, genetic algorithm 
In [18] a network flow based model was given which solves some of the weaknesses of Shapiro's model and is more efficient computationally. Because in our problem the goal is to minimize the number of transactions in a multilateral netting system, there is no linearity of costs, thus neither of these methods can be used as it was briefly discussed in [13].

In [9] the NP-complete Bank Clearing Problem (BCP) was introduced as it occurred in Germany's largest interbank payment system and efficient heuristic algorithms were given to solve it. Later in [17] an approximation algorithm for the $\mathrm{BCP}$ was given. In the $\mathrm{BCP}$ the objective is to maximize the clearing volume with the restriction that the negative net balance cannot exceed a previously deposited amount for each bank. Because the objective and the constraints of the BCP are different from the version of the DC problem discussed here (where the objective is to minimize the number of transactions), these heuristic algorithms cannot be adapted to solve the DC problem and cannot provide a comparison for our proposed evolutionary algorithm.

In [11] a survey is given on solving some other banking related issues (such as portfolio optimization, bankruptcy prediction and FOREX rate prediction) using evolutionary computing. A stochastic model for a payment and settlement system capable of processing payments in real time is presented in [1] by the example of the Clearinghouse of the Bank of Lithuania. Using this model, in [2] several FIFO clearing algorithms are tested by simulation.

The problem of mutual debts compensation (MDC) is formulated using graph theory in $[4,5]$. The author proposes a cycle elimination method, but also shows by an example that the order of elimination is important, which is also mentioned in [13]. In this problem the goal is to maximize the total amount of eliminated debts and can be solved efficiently by linear programming and also by network flow methods. In [6] new models for MDC are introduced.

The problem of settling debts in a minimal number of transactions was discussed by Verhoeff in 2004 ([21]).

Pătcaş [13] later re-discovered the problem and proposed it in 2008 at the qualification contest of the Romanian national team of informatics. The solution was described in [13] and the problem conjectured to be intractable, which was earlier proved in [21]. In [15] the problem's relation to complexity classes was further studied. In [14] the problem in a dynamic setting is discussed and a new algorithm given, having superior speed in some cases compared to the one described in [13]. 
List of borrowings:

\begin{tabular}{|c|c|c|}
\hline Borrower & Lender & Amount of money \\
\hline 1 & 3 & 4 \\
3 & 4 & 7 \\
4 & 2 & 2 \\
2 & 1 & 2 \\
1 & 5 & 1 \\
3 & 5 & 1 \\
5 & 4 & 2 \\
\hline
\end{tabular}

Solution:

\begin{tabular}{|c|c|c|}
\hline Sender & Reciever & Amount of money \\
\hline 1 & 4 & 3 \\
3 & 4 & 4 \\
\hline
\end{tabular}

Figure 1: Example for the DC problem

\section{Stating the problem}

The problem statement is the following $([13])$ :

Let us consider a number of $\mathrm{n}$ entities (persons, companies etc.), and a list of $\mathrm{m}$ borrowings among these entities. A borrowing can be described by three parameters: the index of the borrower entity, the index of the lender entity and the amount of money that was lent. The task is to find a minimal list of money transactions that clears the debts formed among these $\mathrm{n}$ entities as a result of the $\mathrm{m}$ borrowings made.

It is natural to model this problem using graph theory. Consider the following definitions.

Definition 1 ([13]) Let $\mathrm{G}(\mathrm{V}, \mathrm{A}, \mathrm{W})$ be a directed, weighted multigraph without loops, $|\mathrm{V}|=\mathrm{n},|\mathrm{A}|=\mathrm{m}, \mathrm{W}: \mathrm{A} \rightarrow \mathbb{Z}$, where $\mathrm{V}$ is the set of vertices, $\mathrm{A}$ is the set of arcs and $\mathrm{W}$ is the weight function. $\mathrm{G}$ represents the borrowings made, so we will call it the borrowing graph.

The borrowing graph corresponding to the example in Figure 1 is depicted in Figure 2.

Definition 2 ([13]) Let us define for each vertex $v \in V$ the absolute amount of debt over the graph $\mathrm{G}: \mathrm{D}_{\mathrm{G}}(v)=\sum_{\substack{v^{\prime} \in \in \\\left(v^{\prime}, v^{\prime}\right) \in \mathrm{A}}} \mathrm{W}\left(v, v^{\prime}\right)-\sum_{\substack{v^{\prime \prime} \in v \\\left(v^{\prime \prime}, v\right) \in \mathrm{A}}} \mathrm{W}\left(v^{\prime \prime}, v\right)$ 


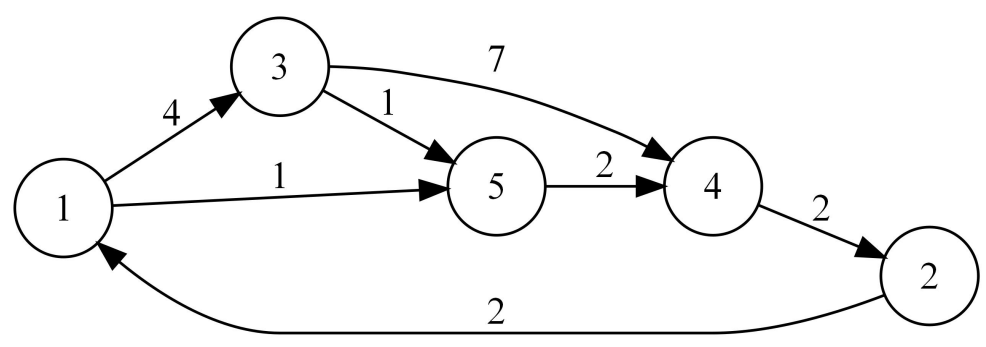

Figure 2: The borrowing graph associated with the given example. An arc from node $i$ to node $j$ with weight $w$ means, that entity $i$ must pay $w$ amount of money to entity $j$.

\begin{tabular}{|c|c|c|c|c|c|}
\hline $\mathfrak{i}$ & 1 & 2 & 3 & 4 & 5 \\
\hline $\mathrm{D}(\mathfrak{i})$ & 3 & 0 & 4 & -7 & 0 \\
\hline
\end{tabular}

Figure 3: Absolute amounts of debt corresponding to the given example.

Sometimes for simplicity we will refer to the absolute amount of debt of a node as D-value.

The D-values corresponding to the example from Figure 1 are listed in Figure 3.

Definition 3 ([13]) Let $\mathrm{G}^{\prime}\left(\mathrm{V}, \mathrm{A}^{\prime}, \mathrm{W}^{\prime}\right)$ be a directed, weighted multigraph without loops, with each arc $(i, j)$ representing a transaction of $W^{\prime}(i, j)$ amount of money from entity $i$ to entity $j$. We call this graph a transaction graph. These transactions clear ${ }^{1}$ the debts formed by the borrowings modeled by graph $\mathrm{G}(\mathrm{V}, \mathrm{A}, \mathrm{W})$ if and only if:

$\mathrm{D}_{\mathrm{G}}\left(v_{\mathrm{i}}\right)=\mathrm{D}_{\mathrm{G}^{\prime}}\left(v_{\mathrm{i}}\right), \forall \mathrm{i}=\overline{1, \mathrm{n}}$, where $\mathrm{V}=\left\{v_{1}, v_{2}, \ldots, v_{\mathrm{n}}\right\}$

We will note this by: $\mathrm{G} \sim \mathrm{G}^{\prime}$.

See Figure 4 for a transaction graph with minimal number of arcs corresponding to the example from Figure 1.

We are now ready to reformulate the problem mathematically:

\footnotetext{
${ }^{1}$ When trying to decide if the transactions described by a transaction graph clear the debts represented by a borrowing graph, it is easy to see that only D-values matter ([21]).
} 

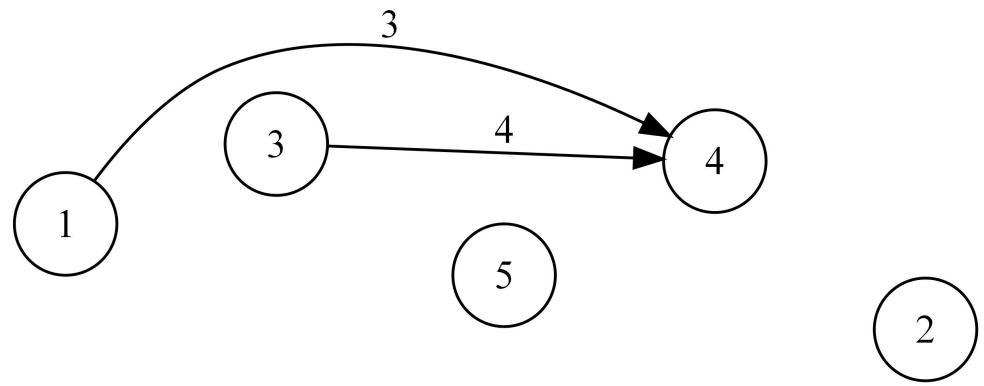

Figure 4: The respective minimum transaction graph. An arc from node $i$ to node $j$ with weight $w$ means, that entity $i$ pays $w$ amount of money to entity j.

Given a borrowing graph $\mathrm{G}(\mathrm{V}, \mathrm{A}, \mathrm{W})$ we are looking for a minimal transaction graph $\mathrm{G}_{\min }\left(\mathrm{V}, \mathrm{A}_{\min }, \mathrm{W}_{\min }\right)$, so that $\mathrm{G} \sim \mathrm{G}_{\min }$ and $\forall \mathrm{G}^{\prime}\left(\mathrm{V}, \mathrm{A}^{\prime}, \mathrm{W}^{\prime}\right)$ : $\mathrm{G} \sim \mathrm{G}^{\prime},\left|A_{\min }\right| \leq\left|\mathrm{A}^{\prime}\right|$ holds.

\section{An equivalent problem}

The following observation is crucial in all of the solutions known so far.

Theorem $4([21,13])$ Any instance of the DC problem can be solved trivially by at most $\mathrm{n}-1$ transactions.

Proof. We give an algorithmic proof.

1. Let us choose two nodes $i$ and $\mathfrak{j}$, such that $\mathrm{D}(\mathfrak{i})>0$ and $\mathrm{D}(\mathfrak{j})<0$.

2. Add arc $(i, j)$ to the transaction graph having weight $\min (D(i),-D(j))$.

3. Update the $\mathrm{D}$-values of $i$ and $j$ to reflect the addition of the arc (by decreasing $D(i)$ and increasing $D(j))$.

4. Repeat steps (1) - (3) as long as possible.

It is clear that at least one D-value becomes zero as a result of executing steps (1) - (3). Also, because we have the invariant that the sum of all Dvalues is always zero, at the last iteration we always have $D(\mathfrak{i})=-D(j)$. Thus 
two D-values become zero at the last iteration, which yields the needed upper bound.

We observe that finding a minimal transaction graph is equivalent to partitioning $\mathrm{V}$ into a maximal number of disjoint zero-sum subsets, more formally $V=P_{1} \cup \ldots \cup P_{\max }, \sum_{u \in P_{i}} D(u)=0, \forall i=\overline{1, \max }$ and $P_{i} \cap P_{j}=\emptyset, \forall i, j=$ $\overline{1, \max }, i \neq j$. The reason for this is, that all the debts in a zero-sum subset $P_{i}$ can be cleared by $\left|P_{i}\right|-1$ transactions by Theorem 4, thus to clear all the debts, $|\mathrm{V}|-$ max transactions are necessary.

\section{Evolutionary technique for solving the DC prob- lem}

We use the reformulation of the problem described in Section 3.

Representation In our method we represent a solution of the problem by a permutation of the $\mathrm{D}$-values of $\mathrm{V}$, the set of nodes. Thus a candidate solution is a vector $C=\left(c_{1}, c_{2}, \ldots, c_{n}\right)$, such that $c_{i}=D(u), \forall i \in \overline{1, n}$ for some unique $\mathrm{u} \in \mathrm{V}$.

For instance $C=(3,0,-7,4,0)$ is a chromosome representing a candidate solution for the D-values from Figure 3.

The idea of representing solutions as permutations was discussed intensively in the context of the Traveling Salesman Problem (TSP) $([7,12,22])$.

Fitness assignment To evaluate the fitness of a chromosome, we need to determine the number of zero-sum subsets codified by the candidate solution, taking into consideration the order of appearance of the D-values. In order to calculate it, we iterate over the genes of the chromosome from left to right and

maintain the partial sum obtained so far, that is $s_{i}=\sum_{j=1}^{i} c_{j}$. For every $s_{i}=0$, we have found a new zero-sum subset of the partition (starting after the last encountered partial sum equal to zero and ending at $i$ ), so we can add one to the fitness of the chromosome.

For instance if we have $C=(-3,2,1,-5,5)$, then $s=(-3,-1,0,-5,0)$, so the fitness of $\mathrm{C}$ will be 2, corresponding to the partition formed by the first three elements and the last two elements. 
Recombination Various operators for permutation representations are discussed in $[3,7,8,12,19,20,22,23]$.

When selecting existing recombination operators or designing new ones the representation of the problem is crucial to consider. In our case we had to take into account that we have a permutation representation corresponding to disjoint sets whose number has to be maximized. Thus for our problem the resulting offsprings of a crossover must represent a valid permutation and must have the potential to improve the number of zero-sum sets.

Because these sets are constructed by looking at the order of genes, for operator Recomb1 we have chosen an order-based crossover method, in particular the Modified Order Crossover (MOX) operator described in [23]. Most crossover operators for permutation representations are designed for the TSP. Replacing a few arcs in the solution of the TSP usually does not greatly change the fitness of the solution, but may have a negative impact in the DC problem by perturbing too many zero-sum sets. Thus we had to be careful which operators to adapt to our problem.

For operator Recomb2 we propose a new crossover operator, which intuitively has a great potential in increasing the number of subsets codified by the offsprings, by leveraging information from the parents.

Recomb1 Let $C_{1}$ and $C_{2}$ be the two chromosomes, and $k \in[1, n]$ a random crossover point. Then, the first descendant $C_{1}^{\prime}$ can be obtained by copying the first $k$ genes from $C_{1}$ and appending to it the elements of the permutation not used so far in the same order as they appear in $C_{2}$. The second descendant $C_{2}^{\prime}$ is obtained symmetrically.

For instance,

$$
\begin{gathered}
k=2 \\
C_{1}=(-3,2,1,-5,5) C_{2}=(-5,2,1,-3,5) \\
\downarrow \\
C_{1}^{\prime}=(-3,2,-5,1,5) C_{2}^{\prime}=(-5,2,-3,1,5)
\end{gathered}
$$

Recomb2 The problem with Recomb1 is, that the first descendant inherits most of its properties from $C_{1}$ and very little from $C_{2}$. Symmetrically $C_{2}^{\prime}$ inherits most of its properties from $C_{2}$ and very little from $C_{1}$. This is undesirable, as both $C_{1}$ and $C_{2}$ can contain subsets from the optimal partition.

A better recombination operator may be the following. First, determine the zero-sum subsets codified by $\mathrm{C}_{1}$ and $\mathrm{C}_{2}$, as described at the fitness assignment. 
Let those be $C_{1}=P_{1,1} \cup P_{1,2} \cup \ldots$ and $C_{2}=P_{2,1} \cup P_{2,2} \cup \ldots$ Initialize $C_{1}^{\prime}:=C_{1}$ and $\mathrm{C}_{2}^{\prime}:=\mathrm{C}_{2}$.

Then, iterate over every $P_{1, i}$. If some $P_{1, i}$ is contained in some $P_{2, j}$, that is $P_{1, i} \subset P_{2, j}$, replace $P_{2, j}$ in the second descendant with $P_{1, i} \cup\left(P_{2, j} \backslash P_{1, i}\right)^{2}$ Repeat the same procedure for $C_{2}$ symmetrically.

For instance,

$$
\begin{gathered}
\mathrm{C}_{1}=(-3,2,1,-5,5)=\{-3,2,1\} \cup\{-5,5\} \\
\mathrm{C}_{2}=(2,1,5,-5,-3)=\{2,1,5,-5,-3\} \\
\downarrow \\
\mathrm{C}_{1}^{\prime}=\{-3,2,1\} \cup\{-5,5\}=(-3,2,1,-5,5) \\
\mathrm{C}_{2}^{\prime}=\{-3,2,1\} \cup\{5,-5\}=(-3,2,1,5,-5)
\end{gathered}
$$

Mutation In our experiments we have used three mutation operators. Mut1 is a classical inversion operator.

We propose two new mutation operators with the property, that the fitness value of the chromosome does not decrease. The new mutation operators are based on Mut1, but are using the additional information of the chosen representation of our particular problem.

Mut1 Holland described an inversion operator in [10], which reverses the order of the elements between two randomly chosen indices. This method can be used without modification, on the sequence between the $i^{\text {th }}$ and $j^{\text {th }}$ elements.

For instance,

$$
\begin{gathered}
i=2, j=5 \\
C=(-3,2, \mathbf{1},-5,5) \\
\downarrow \\
C^{\prime}=(-3,5,-5,1,2)
\end{gathered}
$$

Mut2 Mut1 can be used on the partition $C=P_{1} \cup P_{2} \cup \ldots$ instead of the permutation representation. This method guarantees that the fitness of the chromosome does not decrease.

For instance,

\footnotetext{
${ }^{2}$ In our implementation we have chosen to put the elements of $P_{1, i}$ into $C_{2}^{\prime}$ in the same order as they were in $C_{1}$ and the elements of $P_{2, j} \backslash P_{1, i}$ in the same order as they were in $C_{2}$. Other variations are possible as well.
} 


$$
\begin{gathered}
i=1, j=4 \\
C=(-2,2,3,4,-7,1,-1,6,-3,2,-5)= \\
\{-\mathbf{2}, \mathbf{2}\} \cup\{\mathbf{3}, \mathbf{4},-\mathbf{7}\} \cup\{\mathbf{1},-\mathbf{1}\} \cup\{\mathbf{6},-\mathbf{3}, \mathbf{2},-\mathbf{5}\} \\
\downarrow \\
C^{\prime}=\{\mathbf{6},-\mathbf{3}, \mathbf{2},-\mathbf{5}\} \cup\{\mathbf{1},-\mathbf{1}\} \cup\{\mathbf{3}, \mathbf{4},-\mathbf{7}\} \cup\{-\mathbf{2}, \mathbf{2}\}= \\
(6,-3,2,-5,1,-1,3,4,-7,-2,2)
\end{gathered}
$$

Mut3 Mut1 can also be used inside some $P_{k}$ without decreasing the fitness.

For instance,

$$
\begin{aligned}
& \mathrm{k}=4, \mathrm{i}=1, \mathrm{j}=4 \\
& \mathrm{C}=(-2,2,3,4,-7,1,-1,6,-3,2,-5)= \\
& \{-2,2\} \cup\{3,4,-7\} \cup\{1,-1\} \cup\{\mathbf{6},-\mathbf{3}, \mathbf{2},-\mathbf{5}\} \\
& \downarrow \\
& C^{\prime}=\{-2,2\} \cup\{3,4,-7\} \cup\{1,-1\} \cup\{-\mathbf{5}, \mathbf{2},-\mathbf{3}, \mathbf{6}\}= \\
& (-2,2,3,4,-7,1,-1,-5,2,-3,6)
\end{aligned}
$$

\section{How to obtain large instances of the DC problem}

Because our problem is NP-hard as demonstrated in [15], it is challenging to generate large test cases for which information about the optimal solution is known. We describe five methods to generate large test cases.

Method 1 If the optimal solution for some input is known, padding the set of $\mathrm{D}$-values with $\mathrm{k}$ zeros increases the optimal solution also by $k$.

Method 2 Method 1 can be modified by padding the input with $k$ pairs of the structure $(x,-x)$.

Method 3 If the number of negative (or positive) numbers is two, the problem is equivalent to the Subset Sum problem and is solvable in pseudopolynomial time by dynamic programming. Using this method we can generate inputs for which the optimal solution is unique, that is, there is a single subset of positive (negative) numbers having the sum equal to one of the two negative (positive) numbers (in absolute value). An optimal answer for such an input is expected to be difficult to find for our evolutionary approach, as in the worst case (when the cardinality of the subset is $n / 2$ ) only $2 \cdot\left(\frac{n}{2} !\right)^{2}$ out of the $n$ ! possible permutations do represent an optimal solution. For $n=10$, this 
means that the ratio of optimal solutions and all solutions is about $7.9 \cdot 10^{-3}$, while for $n=100$ the ratio is about $1.9 \cdot 10^{-29}$.

This idea can be extended for any fixed number of negative (positive) numbers, but the running time of the dynamic programming solution raises quickly.

Method 4 Let $n$ be the desired size of the input and $l \leq\lfloor n / 2\rfloor$ an integer. First generate randomly a set of $n-l$ elements, containing only positive $D$ values and $l$ distinct integers from the $[1, n-l]$ range $\left(\right.$ denoted $r_{1}<\ldots<r_{l}$ ).

Let $s$ be the vector of partial sums, that is $s_{i}=\sum_{j=1}^{i} D(j), \forall i=\overline{1, n-l}$ (we

assume $s_{0}=0$ and $\left.r_{0}=0\right)$. For every $r_{i}, \forall i=\frac{j=1}{1, l}$ insert $-\left(s_{r_{i}}-s_{r_{i-1}}\right)$ to the set. In other words we insert with a negative sign the sum of $l$ partial sequences, whose borders are denoted by $r_{i-1}$ and $r_{i}$. By this method we can get the optimal solution to be equal to $l$. As the range of the possible values of the first $n-l$ positive elements gets bigger, we expect the optimal solution to be harder and harder to find. The reason is that the probability to get the same sum from a different combination of positive numbers gets smaller, thus the number of genetic representations corresponding to an optimal solution decreases.

Method 5 It can be easily seen, that if the optimal solution for a set $\mathrm{V}$ is known to be max, then the solution for $\mathrm{V} \cup \mathrm{V}$ will be $2 \cdot \max$, the solution for $\mathrm{V} \cup \mathrm{V} \cup \mathrm{V}$ will be $3 \cdot \max$ and so on.

\section{$6 \quad$ Numerical experiments}

A preliminary testing phase was carried out using the same 15 test cases which were used when the problem was proposed in 2008 at the qualification contest of the Romanian national team (see [14] for a description of each instance). These test cases all have specially crafted structures, with $n \leq 20$, $m \leq 100$ and the cost of an arc being a natural number no larger than 100. For comparison, the optimal solution was determined for each test case by using the algorithm described in [13].

Because these instances have small size, our genetic algorithm can be used with a wide range of parameters and operators to reach the optimal solution in a matter of seconds.

To test the above statement empirically, we used a population of 100 individuals and the number of generations was set 100 . We used operator Recomb2 
in conjunction with roulette wheel selection and operator Mut1 with a mutation probability of 0.5 . The best five individuals always survived to the next generation. Our genetic algorithm found the best solution for all of the test cases.

\subsection{Combinations of operators}

In the first set of experiments our goal was to determine which combinations of our recombination and mutation operators work best in practice, along with desirable values for mutation probability. We constructed three test cases (debt100a, debt100b and debt100c) ${ }^{3}$ with different structures, all of them having $n=100$

debt100a was obtained by concatenating the test case from the initial 15 which was the most difficult to solve for the genetic algorithm (case 15) five times to itself. By the observation above in Method 5, the optimal solution for this test case is $\max =25$.

To generate debt100b we used Method 3 for $n=50$ and concatenated the obtained set once to itself, thus obtaining a case having max $=4$ by the observation above.

To obtain debt100c we first generated, using a dynamic programming algorithm, a set having 20 elements, which can be uniquely partitioned into three zero-sum subsets (and no more). Then we concatenated this set five times to itself, yielding $\max =15$ for this test case.

For each of the three described test cases we used the following methodology. For every possible combination of recombination and mutation operators we fixed the mutation probability to every value from 0 to 1 in steps of 0.1 and executed the genetic algorithm 10 times. We recorded the best solution obtained among the 10 executions, the average of the 10 best values and the average fitness of all genomes. In each case the population size was set to 100 individuals and the number of generations to 1000. For the recombination operators roulette wheel selection was used in every case and the best five individuals always survived to the next generation.

To assess the efficacy of our algorithm we compared it to an algorithm called RandomSearch, which works by generating an independent random solution in every generation for each chromosome. In our case this meant generating 100000 random solutions and remembering the one with the maximum fitness value among them.

\footnotetext{
${ }^{3}$ All test cases used in our experiments can be downloaded from http://cs.ubbcluj.ro/ patcas/debt_experiments.zip
} 
The results of the first set of experiments were the following:

- debt100c was the most difficult of the three test cases used, no algorithm being able to find the optimal solution $\max =15$. The best solution found by RandomSearch was 5 , and the best solution found by the evolutionary algorithms was 13, using Recomb2 along with Mut1 with a mutation probability ranging from 0.8 to 1 . The average fitness of all genomes was maximal at mutation probability 0.7 .

- debt100b was the easiest of the test cases, our genetic algorithm being able to find the optimal solution $\max =4$ in the majority of the cases (in about $76 \%$ of the possible combinations of recombination and mutation operators and mutation probabilities). Mutation probability 0.7 along with Recomb2 and Mut1 maximized the average fitness again. The best solution found by RandomSearch was 3 .

- For debt100a RandomSearch was able to find a solution with fitness 9. Our genetic algorithm found the optimal solution 25 in a small percentage of the cases, using the same parameters that yielded the best solutions for debt100c. Maximal average fitness was obtained with mutation probability 0.4 using Recomb2 and Mut1.

We can draw the conclusion that our genetic algorithm is much more efficient than generating random solutions. The results suggest that using Recomb2 with Mut1 works best in practice for a wide range of inputs. On the other hand we note that Recomb2 and Mut2 is a particularly bad combination, the reason being that it does not allow the exploration of a sufficient varied range of solutions, because neither of the operators is able to introduce new partition sets into the population. Still, Mut2 works fairly well together with Recomb1, as the latter is capable of constructing new partition sets.

\subsection{Convergence to optimum}

In the second set of experiments we studied the convergence of the solution to the optimal value as the number of generations increases. We concatenated each of the three test cases described above ten times to itself, obtaining cases debt1000a, debt1000b and debt1000c respectively. We executed our genetic algorithm using Recomb2 and Mut1 with a mutation probability of 0.75. The population size was set to 80 and the best five individuals were always promoted to the next generation. The algorithm was executed once for 


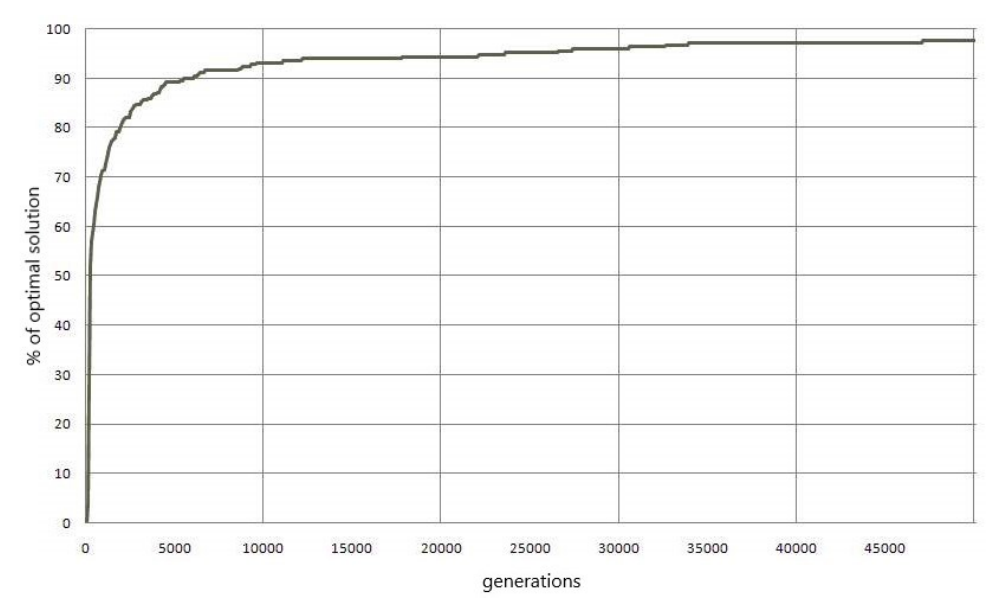

Figure 5: The fitness of the best individual compared to the optimal solution in percentages for test case debt1000a as the number of generations increases.

50000 generations, and the fitness of the best chromosome was recorded every 100 generations.

The results are depicted in Figures 5,6 and 7. We can observe that in every case the fitness of the best individual raises sharply in the first 5000 generations, then slows down gradually. 50000 generations were enough to find a solution having fitness 244 (97.6\% of the optimum) for debt1000a and a solution having fitness 39 (97.5\% of the optimum) for debt1000b. Case debt 1000 c was significantly more difficult, the best solution having only fitness $122(81.3 \%$ of the optimum).

\subsection{Efficiency on very difficult test cases}

In the third set of experiments we used Method 2 to generate test cases which are very difficult for our evolutionary algorithm. Starting with $n=100$ and going by increments of 100 we generated sets having the structure $\{1,2, \ldots, n / 2$, $-1,-2, \ldots,-n / 2\}$. It can be easily seen that the optimal solution for these cases is $\max =n / 2$ and it is unique. Only $\frac{n}{2} ! \cdot 2^{n / 2}$ representations out of $n$ ! translate to an optimal solution, which means that the ratio of optimal solutions to all solutions is about $1.0 \cdot 10^{-3}$ for $n=10$ and about $3.6 \cdot 10^{-79}$ for $n=100$.

For every case we executed the genetic algorithm 10 times using Recomb2 


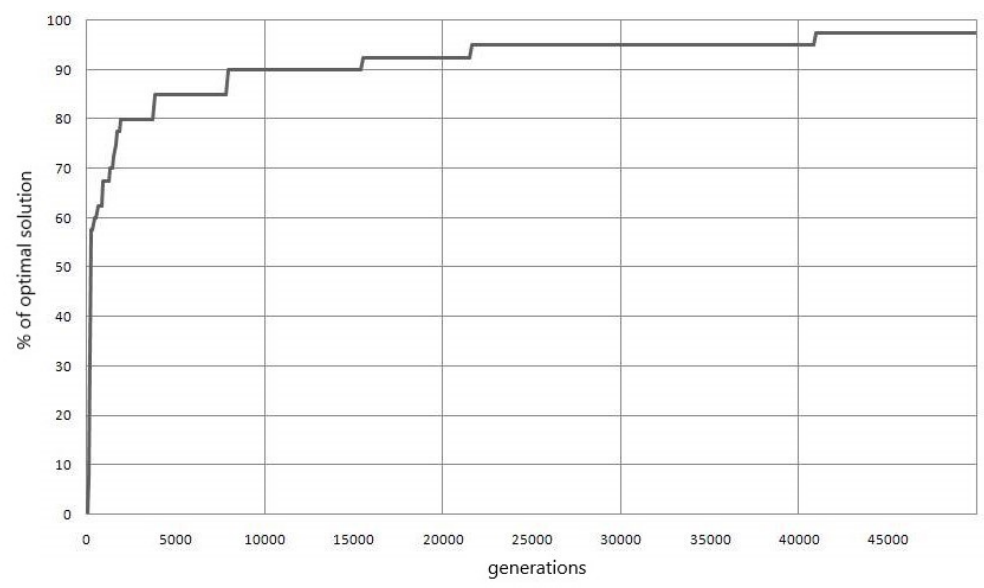

Figure 6: The fitness of the best individual compared to the optimal solution in percentages for test case debt $1000 \mathrm{~b}$ as the number of generations increases.

and Mut1 with a mutation probability 0.75 . The population size was set to 80 and the best five individuals were always promoted to the next generation. The algorithm was stopped after 5000 generations. For every test case we recorded the best solution found by the algorithm, the average of the best solutions over the 10 executions and the summed up running time of the 10 executions. The results are presented in Figure 8.

For $n=100$ the optimal solution was found in all of the 10 executions, but as the size of the input increased, the best solution got further and further from the optimum. We note the robustness of the algorithm, as the best solution is usually just a few percentages away from the average.

\section{Conclusions}

The debts' clearing problem is an NP-hard problem of practical interest, as it arises in real life situations as well. The only known algorithms to solve the problem were the ones presented in [13] and [14], which are exact algorithms that always provide the optimal solution, but their running time is practical only for small inputs $(n \leq 20)$.

Using an equivalent problem, we described an evolutionary algorithm to solve the problem and made extensive experiments to assess its efficacy. From the experiments we concluded that our algorithm is much more efficient than a random search in the space of the solutions. Our algorithm is capable of finding 


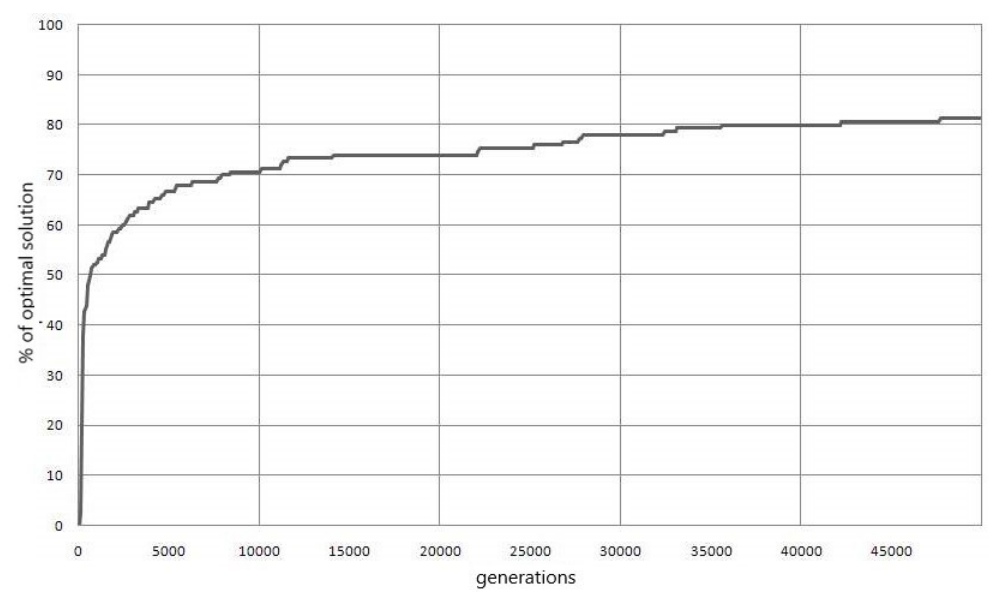

Figure 7: The fitness of the best individual compared to the optimal solution in percentages for test case debt1000c as the number of generations increases.

\begin{tabular}{|c|c|c|c|}
\hline $\mathrm{N}$ & $\begin{array}{c}\text { Best solution } \\
\text { (\% of optimum) }\end{array}$ & $\begin{array}{c}\text { Average of bests } \\
\text { (\% of optimum) }\end{array}$ & $\begin{array}{c}\text { Running time } \\
\text { (in seconds) }\end{array}$ \\
\hline 100 & $50(100 \%)$ & $50(100 \%)$ & 203 \\
200 & $76(76 \%)$ & $70.4(70.4 \%)$ & 710 \\
300 & $91(60.6 \%)$ & $85.5(57 \%)$ & 1247 \\
400 & $108(54 \%)$ & $100.5(50.2 \%)$ & 1919 \\
500 & $116(46.4 \%)$ & $109.8(43.9 \%)$ & 2610 \\
600 & $130(43.3 \%)$ & $121.1(40.3 \%)$ & 3328 \\
700 & $138(39.4 \%)$ & $132(37.7 \%)$ & 4225 \\
800 & $147(36.7 \%)$ & $142.4(35.6 \%)$ & 5134 \\
900 & $155(34.4 \%)$ & $146.6(32.5 \%)$ & 6084 \\
1000 & $166(33.2 \%)$ & $157.3(31.4 \%)$ & 6766 \\
\hline
\end{tabular}

Figure 8: Results of 10 executions for 5000 generations each, on very difficult test cases

the optimal solution for the most difficult test cases with sizes up to $n=100$ in a matter of minutes. For cases as large as $n=1000$ our approach remains practical, as it can obtain solutions in the range of $80 \%-98 \%$ compared to the optimal solution in about an hour on a personal computer. In comparison a random search does not go above $15 \%$ even for the easiest cases of this size. 


\section{References}

[1] D. Bakšys and L. Sakalauskas, Modelling, simulation and optimisation of interbank settlements, Information technology and control 36, 1 (2007) $43-52 . \Rightarrow 143$

[2] D. Bakšys and L. Sakalauskas, Simulation and testing of FIFO clearing algorithms, Information technology and control 39, 1 (2010) 24-31. $\Rightarrow$ 143

[3] L. Davis, Applying adaptive algorithms to epistatic domains, Proceedings of the 9th International Joint Conference on Artificial Intelligence, 162164, Morgan Kaufmann, 1985. $\Rightarrow 148$

[4] V. Gazda, Mutual debts compensation as graph theory application, Challenges for Business Administrators in the New Millennium, 793-811, $2000 . \Rightarrow 143$

[5] V. Gazda, Mutual debts compensation as graph theory problem, Mathematical Finance, 162-167, Springer, 2001. $\Rightarrow 143$

[6] V. Gazda, D. Horváth and M. Rešovskỳ, An application of graph theory in the process of mutual debt compensation, Acta Polytechnica Hungarica 12, 3 (2015) 7-24. $\Rightarrow 143$

[7] D. Goldberg and R. Lingle, Jr. Alleles, loci, and the traveling salesman problem, Proceedings of the 1st International Conference on Genetic Algorithms and their Applications, 154-159, Lawrence Erlbaum Associates, 1985. $\Rightarrow 147,148$

[8] M. Gorges-Schleuter, Genetic algorithms and population structure - A massively parallel algorithm, Ph.D. thesis, University of Dortmund, 1990. $\Rightarrow 148$

[9] M. M. Güntzer, D. Jungnickel and M. Leclerc, Efficient algorithms for the clearing of interbank payments, European Journal of Operational Research 106, 1 (1998) 212-219. $\Rightarrow 143$

[10] J. H. Holland, Adaptation in Natural and Artificial Systems, The University of Michigan Press, $1975 . \Rightarrow 149$

[11] G. J. Krishna and V. Ravi, Evolutionary computing applied to solve some operational issues in banks, Optimization in Industry 31-53, Springer, $2019 . \Rightarrow 143$

[12] I. Oliver, D. Smith and J. Holland, A study of permutation crossover operators on the traveling salesman problem, Proceedings of the Second International Conference on Genetic Algorithms, 224-230, Lawrence Erlbaum Associates, 1987. $\Rightarrow 147,148$ 
[13] C. Pătcaş, On the debts' clearing problem, Studia Universitatis BabeşBolyai Series Informatica, 54, 2 (2009) 109-120. $\Rightarrow 143,144,145,146$, 151,155

[14] C. Pătcaş, The debts' clearing problem: a new approach, Acta Universitatis Sapientiae, Informatica, 3, 2 (2011) 192-204. $\Rightarrow 143,151,155$

[15] C. Pătcaş, The debts' clearing problem's relation with complexity classes, Acta Mathematica Academiae Paedagogicae Nyíregyháziensis, 28, 2 (2012) 217-226. $\Rightarrow 143,150$

[16] A. C. Shapiro, Payments netting in international cash management, Journal of International Business Studies, 9, 2 (1978) 51-58. $\Rightarrow 142$

[17] Y. M. Shafransky and A. A. Doudkin, An optimization algorithm for the clearing of interbank payments, European Journal of Operational Research, 171, 3 (2006) 743-749. $\Rightarrow 143$

[18] V. Srinivasan and Y. H. Kim, Payments netting in international cash management: a network optimization approach, Journal of International Business Studies, 17, 2 (1986) 1-20. $\Rightarrow 142,143$

[19] G. Syswerda, Schedule optimization using genetic algorithms, Handbook of Genetic Algorithms, 332-349, Van Nostrand Reingold, 1991. $\Rightarrow 148$

[20] A. J. Umbarkar and P. D. Sheth, Crossover operators in genetic algorithms: a review, ICTACT journal on soft computing, $6,1,2015 \Rightarrow 148$

[21] T. Verhoeff, Settling multiple debts efficiently: an invitation to computing science, Informatics in Education, 3, 1 (2004), 105-126. $\Rightarrow 143,145,146$

[22] D. Whitley, T. Starkwater and D. Fuquay, Scheduling problems and traveling salesmen: The genetic edge recombination operator, Proceedings of the Third International Conference on Genetic Algorithms, 133-140, Morgan Kaufmann Publishers, 1989. $\Rightarrow 147,148$

[23] J. Wróblewski, Theoretical foundations of order-based genetic algorithms, Fundamenta Informaticae 28, 3-4 (1996) 423-430. $\Rightarrow 148$

Received: September 26, 2019 • Revised: November 26, 2019 